\title{
ARTICLE Bevacizumab or fibronectin gene editing inhibits the osteoclastogenic effects of fibroblasts derived from human radicular cysts
}

\author{
Hai-cheng Wang ${ }^{1}$, Peng Wang ${ }^{2}$, Yuan-wei Chen ${ }^{3}$ and Yan Zhang ${ }^{1}$
}

Fibronectin (FN) is a main component of extracellular matrix (ECM) in most adult tissues. Under pathological conditions, particularly inflammation, wound healing and tumors, an alternatively spliced exon extra domain A (EDA) is included in the FN protein $\left(\mathrm{EDA}^{+} \mathrm{FN}\right)$, which facilitates cellular proliferation, motility, and aggressiveness in different lesions. In this study we investigated the effects of $\mathrm{EDA}^{+} \mathrm{FN}$ on bone destruction in human radicular cysts and explored the possibility of editing FN gene or blocking the related paracrine signaling pathway to inhibit the osteoclastogenesis. The specimens of radicular cysts were obtained from 20 patients. We showed that the vessel density was positively associated with both the lesion size $(R=0.49, P=0.001)$ and EDA ${ }^{+} \mathrm{FN}$ staining $(R=0.26, P=0.022)$ in the specimens. We isolated fibroblasts from surgical specimens, and used the CRISPR/Cas system to knockout the EDA exon, or used IST-9 antibody and bevacizumab to block EDA ${ }^{+}$FN and VEGF, respectively. Compared to control fibroblasts, the fibroblasts from radicular cysts exhibited significantly more Trap ${ }^{+} \mathrm{MNCs}$, the relative expression level of VEGF was positively associated with both the ratio of $\mathrm{EDA}^{+} \mathrm{FN} /$ total FN $(R=0.271, P=0.019)$ and with the number of Trap ${ }^{+} \mathrm{MNCs}(R=0.331$, $P=0.008$ ). The knockout of the EDA exon significantly decreased VEGF expression in the fibroblasts derived from radicular cysts, leading to significantly decreased osteoclastogenesis; similar results were observed using bevacizumab to block VEGF, but block of $\mathrm{EDA}^{+} \mathrm{FN}$ with IST-9 antibody had no effect. Furthermore, the inhibitory effects of gene editing on Trap ${ }^{+}$MNC development were restored by exogenous VEGF. These results suggest that EDA ${ }^{+} F N$ facilitates osteoclastogenesis in the fibrous capsule of radicular cysts, through a mechanism mediated by VEGF via an autocrine effect on the fibroblasts. Bevacizumab inhibits osteoclastogenesis in radicular cysts as effectively as the exclusion of the EDA exon by gene editing.

Keywords: odontogenic cysts; radicular cysts; osteoclastogenesis; EDA ${ }^{+}$FN; VEGF; gene editing; bevacizumab; IST-9 antibody

Acta Pharmacologica Sinica (2019) 40:949-956; https://doi.org/10.1038/s41401-018-0172-x

\section{INTRODUCTION}

As the main component of the extracellular matrix (ECM), fibronectin (FN) is important and is widely distributed in various tissues. Despite its absence in most adult tissues, the alternatively spliced exon extra domain $\mathrm{A}(\mathrm{EDA})$ is included in the $\mathrm{FN}$ protein $\left(\mathrm{EDA}^{+} \mathrm{FN}\right)$ under pathological conditions, particularly inflammation, wound healing and tumors [1, 2]. This protein facilitates cellular proliferation, motility, and aggressiveness in different lesions $[3,4]$. Although the effects of $\mathrm{EDA}^{+} \mathrm{FN}$ on bone destruction remain unclear, the peptide motif (CTYSSPEDGIHEC) within the EDA fragment exhibits affinity for integrins [5] or Toll-like receptors (TLRs) [6] in preosteoclasts. In addition, ligand-receptor interactions in stromal cells may lead to paracrine induction of osteoclastogenesis-related genes, including TNF- $a$, IL-6, IL-1 $\beta$, and VEGF $[6,7]$.

In a previous study, the EDA exon maintained VEGF expression and in turn ensured the vascularization and dissemination of tumors, thereby contributing to tumor aggressiveness [2]. We have previously investigated hyperplastic vessels in odontogenic cysts [8] and the osteoclastogenesis induced by VEGF [9], since this growth factor sometimes induces osteoclastogenesis as a substitute for macrophage colony-stimulating factor (M-CSF) $[10,11]$. In the stroma of odontogenic cysts, signaling from $\mathrm{EDA}^{+} \mathrm{FN}$ to VEGF may participate in subsequent osteoclastogenesis and further enhance the characteristic bone destruction. As the most prevalent odontogenic cysts, radicular cysts comprise approximately half of the incidence [12]; chronic inflammation in the fibrous capsules surrounding the destroyed bone cavity tends to promote EDA ${ }^{+} F N$ expression $[1,13]$. Then, VEGF expression is induced to facilitate vascularization and the subsequent infiltration of osteoclast precursors [14].

Therefore, in the present study, the associations among $\mathrm{EDA}^{+} \mathrm{FN}$, vessels, and bone destruction in radicular cysts were investigated. Bevacizumab [15] or the type II bacterial clustered, regularly interspaced, palindromic repeats (CRISPR)-associated (Cas) system

\footnotetext{
${ }^{1}$ Department of Pathology, School \& Hospital of Stomatology, Tongji University, Shanghai Engineering Research Center of Tooth Restoration and Regeneration, Shanghai 200072, China; ${ }^{2}$ State Key Laboratory of Oral Diseases, Dept. of Cleft Lip and Palate Surgery, West China Hospital of Stomatology, Sichuan University, Chengdu 610041, China and

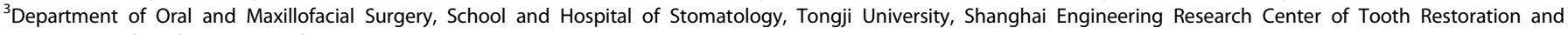
Regneration, Shanghai 200072, China

Correspondence: Yan Zhang (zhangyankq@tongji.edu.cn)

These authors contributed equally: Hai-cheng Wang, Peng Wang
}

Received: 28 May 2018 Accepted: 14 September 2018

Published online: 31 October 2018 


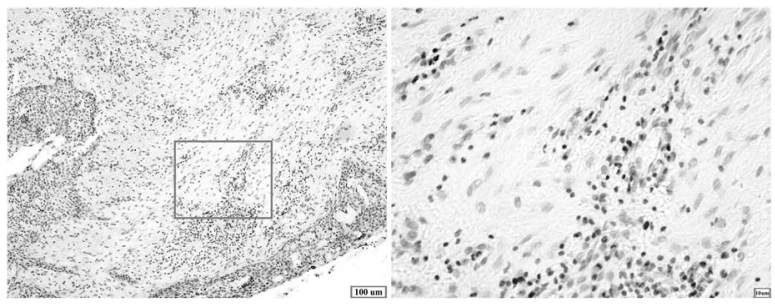

Low expression

EDA $^{+}$FN

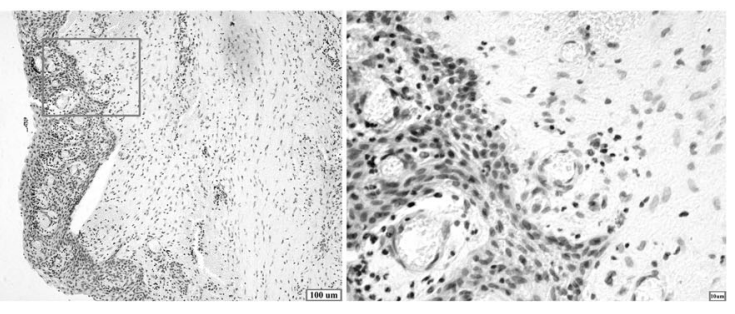

High expression b

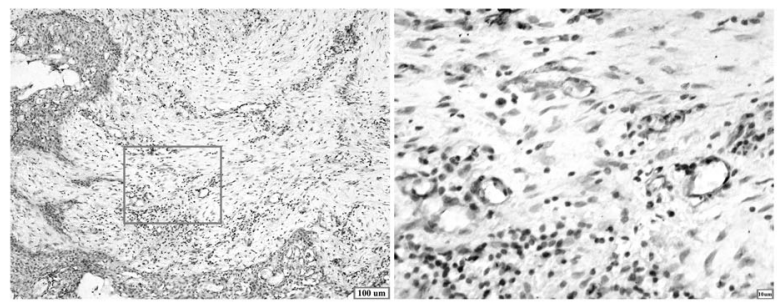

Low density

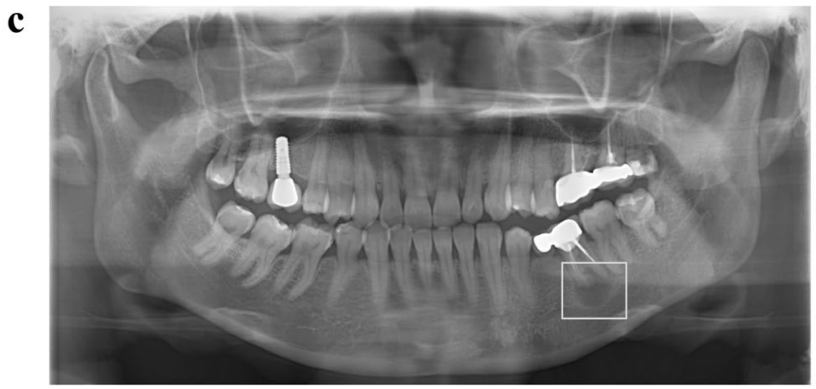

$\mathrm{CD34}^{+}$vessels

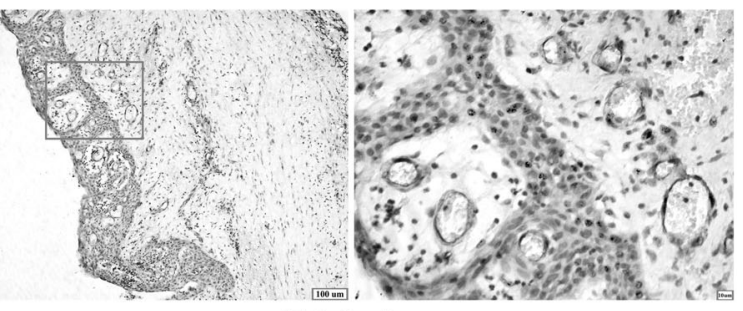

High density

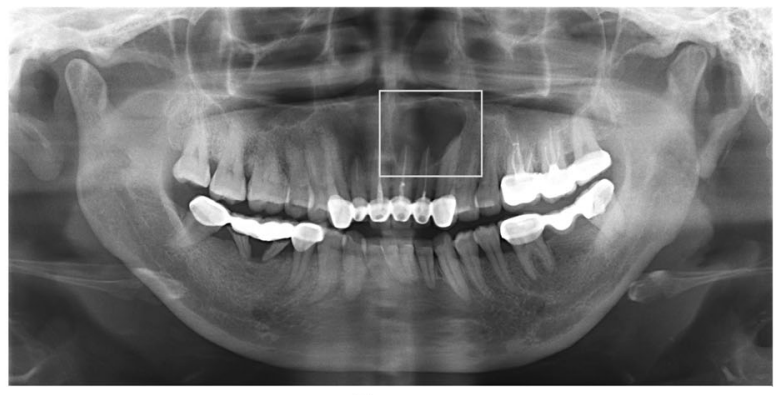

Large

Fig. 1 a Evaluation of the immunohistochemical staining of EDA ${ }^{+} F N$ in the stroma of radicular cysts, by demarcating high or low expression groups (original magnification $\times 100$; local magnification $\times 400$ ). $\mathbf{b}$ Evaluation of the areas of microvessels by demarcating the groups of high or low density (original magnification $\times 100$; local magnification $\times 400$ ). $c$ Evaluation of the radiolucent areas of radicular cysts by demarcating the groups of large or small areas

$[2,4]$ was used to block the effects of either VEGF or EDA ${ }^{+} \mathrm{FN}[16]$, respectively, and inhibit osteoclastogenesis.

\section{MATERIALS AND METHODS}

The standard for selection of patients with radicular cysts All patients were surgically treated at the Department of Oral and Maxillofacial Surgery, TongJi University School of Stomatology, from 2016 to 2017, and were diagnosed radicular cysts by the Shanghai Fudan Clinical Diagnostic Pathology Center. Clinical information, including age, gender, and radiographs, was collected from case histories and follow-up data. The period between acute periapical periodontitis and the first visit was no more than one year. All patients provided written informed consent for the use of their lesion samples for research purposes, as approved by the Review Board of TongJi University.

Immunohistochemical (IHC) investigation of the specimens The formalin-fixed, paraffin-embedded radicular cyst specimens were obtained from the Shanghai Fudan Clinical Diagnostic Pathology Center. The specimens were sectioned into 4- $\mu \mathrm{m}$-thick slices and stained with an IST-9 and anti-CD34 antibody (Abcam Ltd., Cambridge, MA, USA) at $4{ }^{\circ} \mathrm{C}$ overnight to investigate EDA ${ }^{+} \mathrm{FN}$ and the endothelia, respectively. Phosphate-buffered saline (PBS) was used as the negative control. The slices were subsequently incubated with a biotinylated secondary antibody (1:200) for $1 \mathrm{~h}$. Immunocomplexes were visualized with diaminobenzidine
(Zhongshan Golden Bridge Biological Technology Co., Ltd., Beijing, China).

As described in a previous study [17], EDA ${ }^{+} \mathrm{FN}$ staining in the stroma was quantified using a microscope (Olympus, Tokyo, Japan) and the Image-Pro Plus ver. 6.0 software (Media Cybernetics, Silver Spring, MD, USA). The optical density was calibrated, and the area of interest was set as follows: hue, 0-30; saturation, 0-255; and intensity, 0-255. Then, the median of the integrated optical density (IOD) in the stromal area $\left(\mathrm{IOD} / \mu \mathrm{m}^{2}\right)$ was used to demarcate the groups with high or low expression (Fig. 1a). According to previous studies [8, 18], the areas surrounded by the $\mathrm{CD}_{3} 4^{+}$vascular endothelia in the adjacent connective tissue within a depth of $500 \mu \mathrm{m}$ from the lining epithelium were measured as microvessels, and the median area $\left(\mu \mathrm{m}^{2}\right)$ in each randomly selected high-power field (hpf: 400x magnification) was used to demarcate the groups displaying a high or low density (Fig. 1b).

Imaging and measurement of the panoramic radiograph The digital panoramic radiographs were taken with the PM 2002 CC Proline (Planmeca OY, Helsinki, Finland) (filtration: $2.5 \mathrm{~mm}$ AlEquiv) using Kodak T-MAT G/RA Dental Film (Eastman Kodak, Rochester, NY, USA). The magnification factor varied from a maximum of 1.30 in the incisor region to a minimum of 1.25 at the condyle as described previously [19]. The borders of the lesions were determined by an observer after tracing the outlines using the Image-Pro Plus ver. 6.0 software. The median areas $\left(\mathrm{mm}^{2}\right)$ 
Table 1. Sequences of CRISPR sgRNA and confirming primers used in this study

\begin{tabular}{|c|c|c|c|}
\hline Name & sgRNA sequence $\left(5^{\prime}-3^{\prime}\right)$ & PAM sequences $\left(5^{\prime}-3^{\prime}\right)$ & DSB site in fibronectin (FN) genome (ref|NC_018913.2|) \\
\hline sgRNA upstream-R & AACTTAGGGCGATCAATGTCTGT & & \\
\hline \multirow[t]{2}{*}{ sgRNA downstream-R } & AACGTGGA CTGGGTTCCAATCAG & & \\
\hline & Primers & Product containing EDA & Product without EDA \\
\hline
\end{tabular}

were used to demarcate the groups with large or small radiolucent areas (Fig. 1c).

Cell culture

Fresh specimens were obtained after surgery from all included patients who were admitted to the TongJi University Hospital of Stomatology under institutionally approved protocols after obtaining informed consent. Fibroblasts were isolated from the stroma and cultured in a-modified Eagle's medium (a-MEM) (Gibco, Grand Island, NY, USA), as described in our previous studies $[9,20]$. Jaw bone fibroblasts from 6 patients who underwent orthognathic surgery were used as controls.

The Raw264.7 murine osteoclast precursor cell line was routinely cultured in Dulbecco's modified Eagle's medium (DMEM) (Gibco). The medium contained $1 \%$ antibiotics $(100 \mathrm{U} / \mathrm{mL}$ of penicillin and $100 \mathrm{~g} / \mathrm{mL}$ of streptomycin), $2 \mathrm{mM} \mathrm{L-glutamine,} \mathrm{and}$ $10 \%$ fetal bovine serum (FBS). All cells were routinely cultured at $37^{\circ} \mathrm{C}$ with $95 \%$ humidity and a $5 \% \mathrm{CO}_{2}$ atmosphere.

Collection of conditioned medium from fibroblasts and induction of osteoclastogenesis

Fibroblasts were seeded onto $100-\mathrm{mm}$ dishes and grown to $70 \%-80 \%$ confluence. Then, the medium was replaced with serum-free a-MEM and maintained for 7 days without change. As described previously [20], the supernatant was collected, centrifuged at $550 \times g$ for $10 \mathrm{~min}$, aliquoted, and stored at $20^{\circ} \mathrm{C}$. Then, a mixture comprising $50 \%$ supernatant, $40 \%$ fresh a-MEM (GIBCO), $10 \%$ FBS and $12 \mathrm{ng} / \mu \mathrm{L}$ of recombinant murine RANKL (R\&D Systems, Minneapolis, MN, USA) was prepared to generate conditioned medium and induce osteoclasts in vitro.

Raw264.7 cells were seeded into 24 -well plates at a density of 1000 cells/well and cultured with the conditioned medium. The medium was replaced every 2 days for 10 days. The cells were stained for tartrate-resistant acid phosphatase (Trap) (Trap kit; Sigma, St. Louis, MO, USA) according to the manufacturer's protocol. Trap-positive cells containing no fewer than three nuclei $\left(\operatorname{Trap}^{+} \mathrm{MNCs}\right)$ were counted as osteoclast-like cells.

Knockout of the EDA exon and modulation of the conditioned medium

As described in our previous studies [2, 4], two sgRNAs were designed to complement the upstream (sgRNA-upstream) or downstream (sgRNA-downstream) region of the EDA exon at sites with a PAM sequence; oligo DNA sequences encoding sgRNAs were individually annealed and cloned into the CRISPR/Cas9 plasmid PX330. Then, the two sgRNA-guided CRISPR/Cas9 plasmids were confirmed by DNA sequencing. The medium was replaced with serum-free medium, and the cells were incubated for $6 \mathrm{~h}$ until they reached $\sim 70 \%-90 \%$ confluence. The two sgRNA-guided CRISPR/ Cas9 plasmids were cotransfected with Lipofectamine 2000 (Life Technologies, Carlsbad, CA, USA). Thirty-six hours later, the medium was replaced with medium containing puromycin and incubated for at least $24 \mathrm{~h}$. After replacement with fresh medium and culture for an additional $24 \mathrm{~h}$, the surviving cells were collected and transferred to new dishes. When the confluence was $>50 \%$, the supernatant was collected for the conditioned medium as described above. Genomic DNA was extracted to assess the efficiency of the EDA knockout using PCR, and the EDA-negative FN gene was confirmed by DNA sequencing. The sequences of the confirmation primers and sgRNAs are identical to those described in our previous study [4] (Table 1).

The IST-9 antibody (Abcam) and bevacizumab (Genentech Inc., South San Francisco, CA, USA), which were designed to specifically block $\operatorname{EDA}^{+} \mathrm{FN}[21]$ and $\operatorname{VEGF}[22,23]$, respectively, and recombinant human VEGF (R\&D Systems, Minneapolis, MN, USA) were added to the conditioned medium according to the VEGF protein levels in the conditioned medium. An enzymelinked immunosorbent assay (ELISA) kit (Boster Biological Technology, Wuhan, China) was used to measure the VEGF protein levels in the supernatant according to the manufacturer's instructions.

RNA extraction, reverse transcription, and PCR amplification Total RNA was isolated from all cells with the TRIzol Reagent (Life Technologies). First, $2 \mu \mathrm{g}$ of total RNA was reversetranscribed into CDNA using the Superscript First-Strand Synthesis System (Life Technologies) according to the manufacturer's instructions. The reactions were conducted in a $20-\mu \mathrm{L}$ reaction mixture with the LightCycler Real-Time PCR System (Roche Diagnostics Ltd., Shanghai, China), including an initial incubation at $95^{\circ} \mathrm{C}$ for $10 \mathrm{~min}$, followed by 40 cycles of annealing/extension at $60^{\circ} \mathrm{C}$ for $1 \mathrm{~min}$ and denaturation at $95^{\circ} \mathrm{C}$ for $15 \mathrm{~s}$ [9]. The expression of the FN isoforms and genes related to osteoclastogenesis was normalized to human $\beta$-actin expression as described previously [4]. The primers are described in Table 2.

\section{Statistics}

The Chi-square test was used to evaluate $\mathrm{EDA}^{+} \mathrm{FN}$ staining in the different groups stratified by clinical characteristics. The associations among the IOD, areas, $\operatorname{Trap}^{+} \mathrm{MNCs}$ and relative gene expression were analyzed with univariate logistic regression and Spearman's rank correlation analyses. Quantitative data were presented as the means \pm standard deviations (SDs) and were analyzed using Student's $t$-test to determine differences between paired groups. Statistical significance was set to $P<0.05$. Each experiment was performed in triplicate.

\section{RESULTS}

Histological and radiographic investigations of radicular cysts The specimens exhibited a stratified squamous epithelial lining surrounded by fibrous capsules (Fig. 1a, b), which was consistent 
Table 2. The primers used for real-time PCR

\begin{tabular}{llll}
\hline Fibronectin isoforms (NM_212482) & Forward primers $\left(5^{\prime}-3^{\prime}\right)$ & Reverse primers $\left(5^{\prime}-3^{\prime}\right)$ & Sites of amplification \\
\hline EDA $^{+}$FN (NM_212482) & AGGACTGGCATTCACTGATGTG & GTCACCCTGTACCTGGAAACTTG & $5447-5533$ \\
Total FN (NM_212482) & GTGCCACTTCCCCTTCCTAT & ATCCCACTGATCTCCAATGC & $1523-1721$ \\
VEGF (NM_001171623.1) & TTATGCGGATCAAACCTCACC & GAAGCTCATCTCTCCTATGTGC & $1355-1404$ \\
\hline
\end{tabular}

\begin{tabular}{|c|c|c|c|c|}
\hline Parameters & $\begin{array}{l}\text { Low EDA }{ }^{+} \mathrm{FN} \\
\text { expression }\end{array}$ & $\begin{array}{l}\text { High } \mathrm{EDA}^{+} \mathrm{FN} \\
\text { expression }\end{array}$ & $\chi^{2}$ value & $P$-value \\
\hline \multicolumn{5}{|l|}{ Age (year) } \\
\hline$\leq 50$ & $4(40 \%)$ & $6(60 \%)$ & 0.8 & 0.371 \\
\hline$>50$ & $6(60 \%)$ & $4(40 \%)$ & & \\
\hline \multicolumn{5}{|l|}{ Gender } \\
\hline Male & $5(45.5 \%)$ & $6(54.5 \%)$ & 0.202 & 0.653 \\
\hline Female & $5(55.6 \%)$ & $4(44.4 \%)$ & & \\
\hline \multicolumn{5}{|c|}{ The size of lesion $\left(\mathrm{mm}^{2}\right)$} \\
\hline$\leq 150$ & $8(80.0 \%)$ & $2(20.0 \%)$ & 7.2 & $* 0.023$ \\
\hline$>150$ & $2(20.0 \%)$ & $8(80.0 \%)$ & & \\
\hline \multicolumn{5}{|c|}{ Period (month) } \\
\hline$\leq 5.5$ & $4(40 \%)$ & $6(60 \%)$ & 0.8 & 0.371 \\
\hline$>5.5$ & $6(60 \%)$ & $4(40 \%)$ & & \\
\hline \multicolumn{5}{|l|}{ Region } \\
\hline Maxillary & $2(33.3 \%)$ & $4(66.7 \%)$ & 0.952 & 0.329 \\
\hline Mandible & $8(57.1 \%)$ & $6(42.9 \%)$ & & \\
\hline
\end{tabular}

with the histological characteristics of radicular cysts [24]. The radiolucent areas surrounding the apex of the tooth in the panoramic radiograph exhibiting defined borders were selected for this study (Fig. 1c).

Consistent with the total FN staining [25], immunohistochemical staining for $\mathrm{EDA}^{+} \mathrm{FN}$ exhibited a diffuse pattern in both the fibrous capsule and the epithelial lining (Fig. 1a), and the microvessel areas were surrounded by CD34-stained vascular endothelia as described previously [2] (Fig. 1b). EDA ${ }^{+} F N$ staining in the fibrous capsule tended to appear in patients with large lesions (Table 3 ) (Fig. 1c). In the adjacent connective tissue within a depth of 500 $\mu \mathrm{m}$ from the epithelium $[8,18]$, the vessel density $\left(\mu \mathrm{m}^{2}\right.$, vessel areas per $\mathrm{hpf}$ ) was positively associated with both the radiolucent areas $(n=20, R=0.490, P=0.001)$ and $\mathrm{EDA}^{+} \mathrm{FN}$ staining $(n=20$, $R=0.260, P=0.022$ ), which was consistent with the Spearman's rank correlation analysis results $(P=0.015$ and $P=0.050$, respectively) (Fig. 2a, b). These results suggest that EDA ${ }^{+} F N$ exerts effects on VEGF, since VEGF mainly contributes to vascularization [26].

The relationships among the EDA ${ }^{+} \mathrm{FN} /$ total FN ratio, VEGF expression, and osteoclastogenesis

The $\mathrm{EDA}^{+} \mathrm{FN}$ and VEGF mRNA levels were assessed in fibroblasts obtained from cysts and control tissues. Although the differences in the relative expression of VEGF were not significant, the fibroblasts from the cysts displayed a significantly higher $\mathrm{EDA}^{+} \mathrm{FN} /$ total FN ratio $(P=0.003)$ (Fig. $2 \mathrm{c}$ ). This ratio was also positively associated with relative VEGF expression, as illustrated by the univariate logistic regression analysis $(n=20, R=0.271, P=0.019)$ (Fig. 2d) and Spearman's rank correlation analysis $(P<0.001)$.
Consistently, the fibroblasts from the cysts induced significantly more $\mathrm{Trap}^{+} \mathrm{MNCs}$ than the control fibroblasts $(12.075 \pm 7.50$ vs $5.29 \pm 2.17, P=0.041)$ (Fig. $2 \mathrm{e}, \mathrm{f})$, and the relative VEGF expression in the fibroblasts from the cysts was positively associated with the $\operatorname{Trap}^{+} \mathrm{MNC}$ numbers $(n=20, R=$ $0.331, P=0.008$ ) (Fig. $2 \mathrm{~g}$ ). Spearman's rank correlation analysis also suggested a positive correlation $(P=0.001)$. Thus, osteoclastogenesis induced by fibroblasts derived from cysts might be attributed to not only $\mathrm{EDA}^{+} \mathrm{FN}$ itself but also to paracrine VEGF signaling through a mechanism dependent on alternative splicing of the EDA exon [2].

The effect of exclusion of the alternatively spliced EDA exon The EDA exon was specifically knocked out from the FN gene in fibroblasts from cysts using the CRISPR/Cas system. PCR products lacking the EDA exon were observed as a 415 base pair (bp) band, whereas the EDA-containing product had a length of $675 \mathrm{bp}$; both bands were observed in the EDA-knockout lane, suggesting exclusion of the EDA exon in some cells (Fig. 3a). According to the DNA sequencing data, the double-strand breaks (DSBs) were repaired by non-homologous end-joining (NHEJ) (Fig. 4b), which was consistent with the results from a previous study [4]. Although the total FN level remained stable, the $\mathrm{EDA}^{+} \mathrm{FN}$ protein level was noticeably decreased compared with that of the untreated cells (Fig. 3c); in addition, significant decreases in both the VEGF protein and mRNA levels $(0.00797 \pm 0.00503$ vs $0.00251 \pm 0.00126$, $P=0.027$ ) were observed in cells lacking the EDA exon (Fig. 3c, d) (Table 4).

The effects of EDA blockade on osteoclastogenesis

In addition to the EDA-knockout group, IST-9 was used to block the interaction between $\mathrm{EDA}^{+} \mathrm{FN}$ and preosteoclasts $\left(\mathrm{EDA}^{+} \mathrm{FN}\right.$ blocked group), and bevacizumab was used to block the effects of VEGF (bevacizumab group). Conversely, exogenous recombinant human VEGF (rhVEGF) was used to restore the osteoclastogenic effects of the EDA-knockout cells (EDA knockout group and EDA knockout+rhVEGF group). Conditioned medium from untreated fibroblasts from cysts was used as a control (Fig. 4a).

The numbers of $\mathrm{Trap}^{+} \mathrm{MNCs}$ that developed in the untreated and $\mathrm{EDA}^{+} \mathrm{FN}$ blocked group were not significantly different $(n=6$, $8.792 \pm 1.833$ vs $9.292 \pm 1.933, P=0.656)$, suggesting that $\mathrm{EDA}^{+} \mathrm{FN}$ and preosteoclasts were not directly correlated. In contrast to the untreated controls, the number of $\mathrm{Trap}^{+} \mathrm{MNCs}$ was significantly decreased to approximately similar levels in the EDA knockout $(n=6,6.083 \pm 2.217, P=0.011)$ and bevacizumab groups $(n=6$, $6.375 \pm 1.438, P=0.029$ ). However, in the EDA-knockout cells, rhVEGF treatment significantly restored the number of $\mathrm{Trap}^{+} \mathrm{MNCs}$ $(n=6,8.500 \pm 1.304, P=0.044)$ to a significantly greater number than that in the bevacizumab group $(n=6, P=0.023)$; the value was similar to that of the untreated controls (Fig. 4b). Thus, VEGF was primarily responsible for the osteoclastogenesis induced by $E D A^{+} F N$ in fibroblasts from radicular cysts.

\section{DISCUSSION}

A radicular cyst is a typical odontogenic cyst that results from chronic periapical periodontitis [24]. The inflammatory stroma 

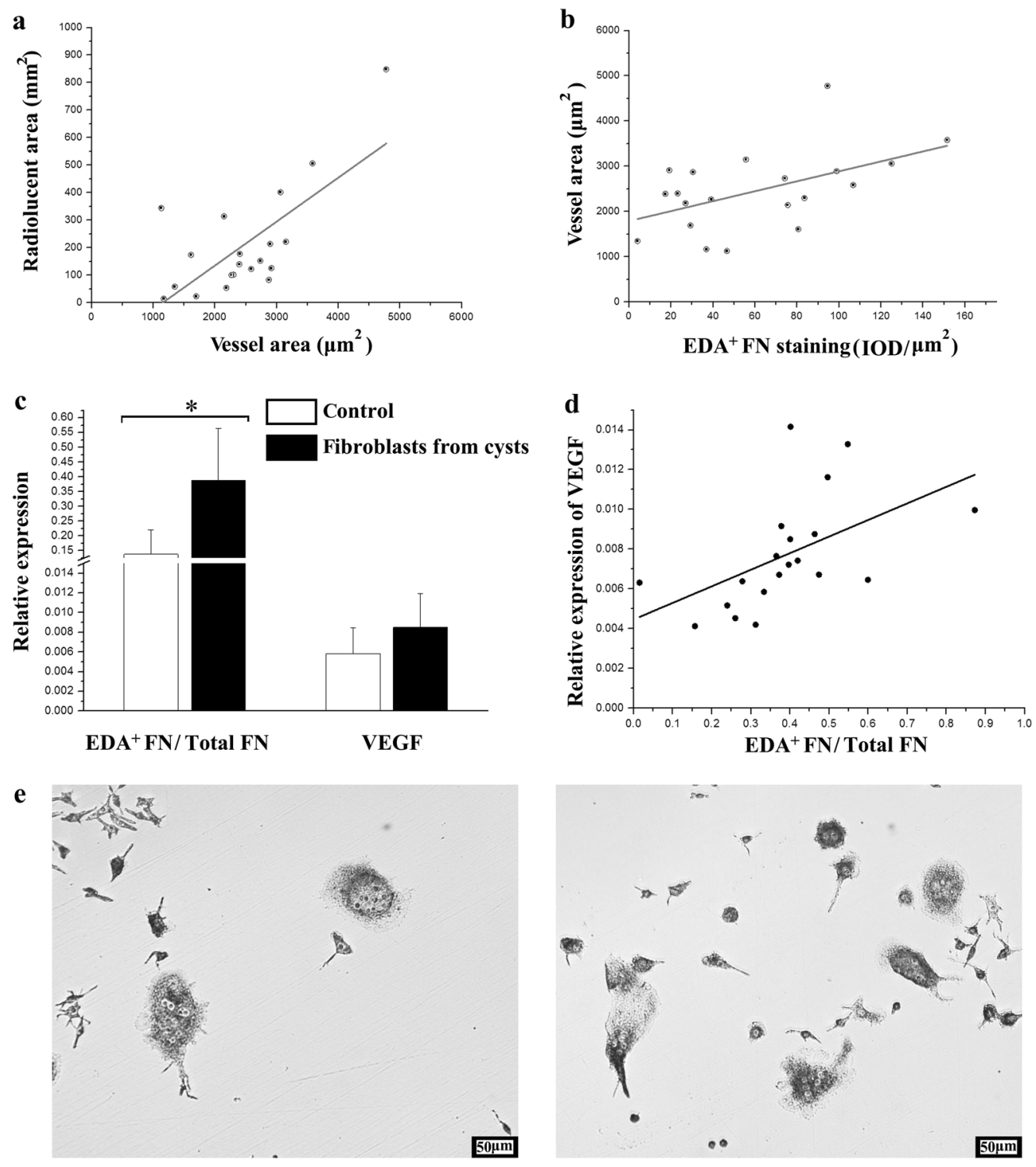

Control

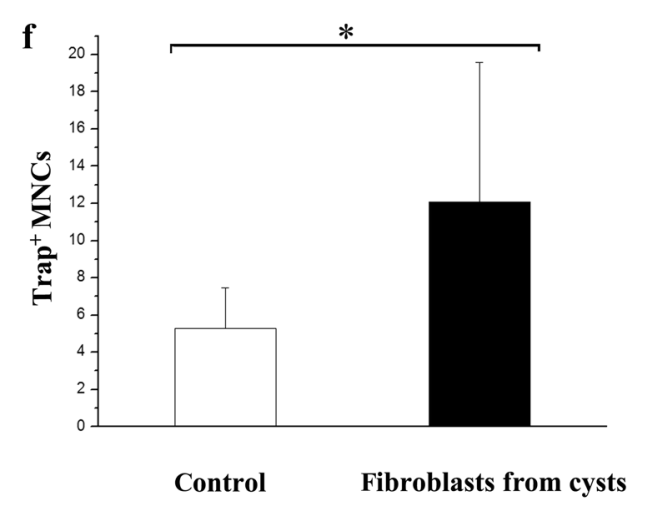

Fibroblasts from cysts

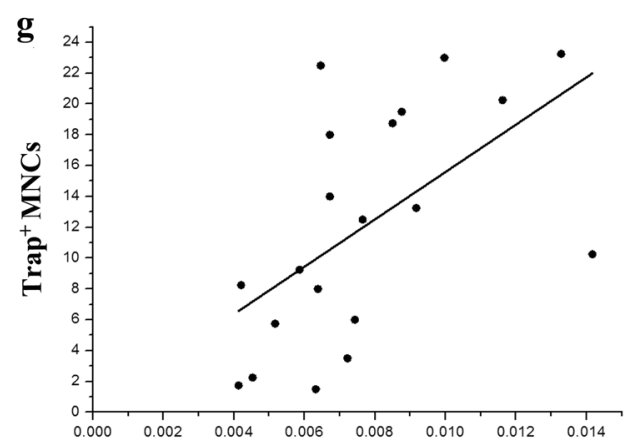

Relative expression of VEGF

Fig. 2 a The positive association between the radiolucent areas and vessel areas per high-power field (hpf) $(n=20, \gamma=0.490, P=0.001)$. b The positive association between the staining of EDA ${ }^{+} \mathrm{FN}$ and vessel areas/hpf in the stroma of radicular cysts $(n=20, \gamma=0.260, P=0.022)$. $\mathrm{C}$ The comparison of the ratio of EDA ${ }^{+} \mathrm{FN} /$ total FN as well as the relative expression of VEGF, between the fibroblasts isolated from cysts or the jaw bone (control). d The positive association of the ratio of EDA ${ }^{+} \mathrm{FN} /$ total FN with the relative expression of VEGF, in the fibroblasts isolated from cysts $(n=20, \gamma=0.271, P=0.019)$. e The Trap ${ }^{+}$MNCs developed from the conditioned medium (original magnification $\times 200$, scale bar: $50 \mu \mathrm{m})$. f The comparison of the Trap ${ }^{+} \mathrm{MNCs}$ developed in the conditioned medium made from fibroblasts from cysts or controls. $\mathbf{g}$ The positive association between the relative expression of VEGF in the fibroblasts from cysts, as well as the Trap ${ }^{+}$MNCs developed in the conditioned medium $(n=20, \gamma=0.331, P=0.008)\left({ }^{*} P<0.05\right)$ 

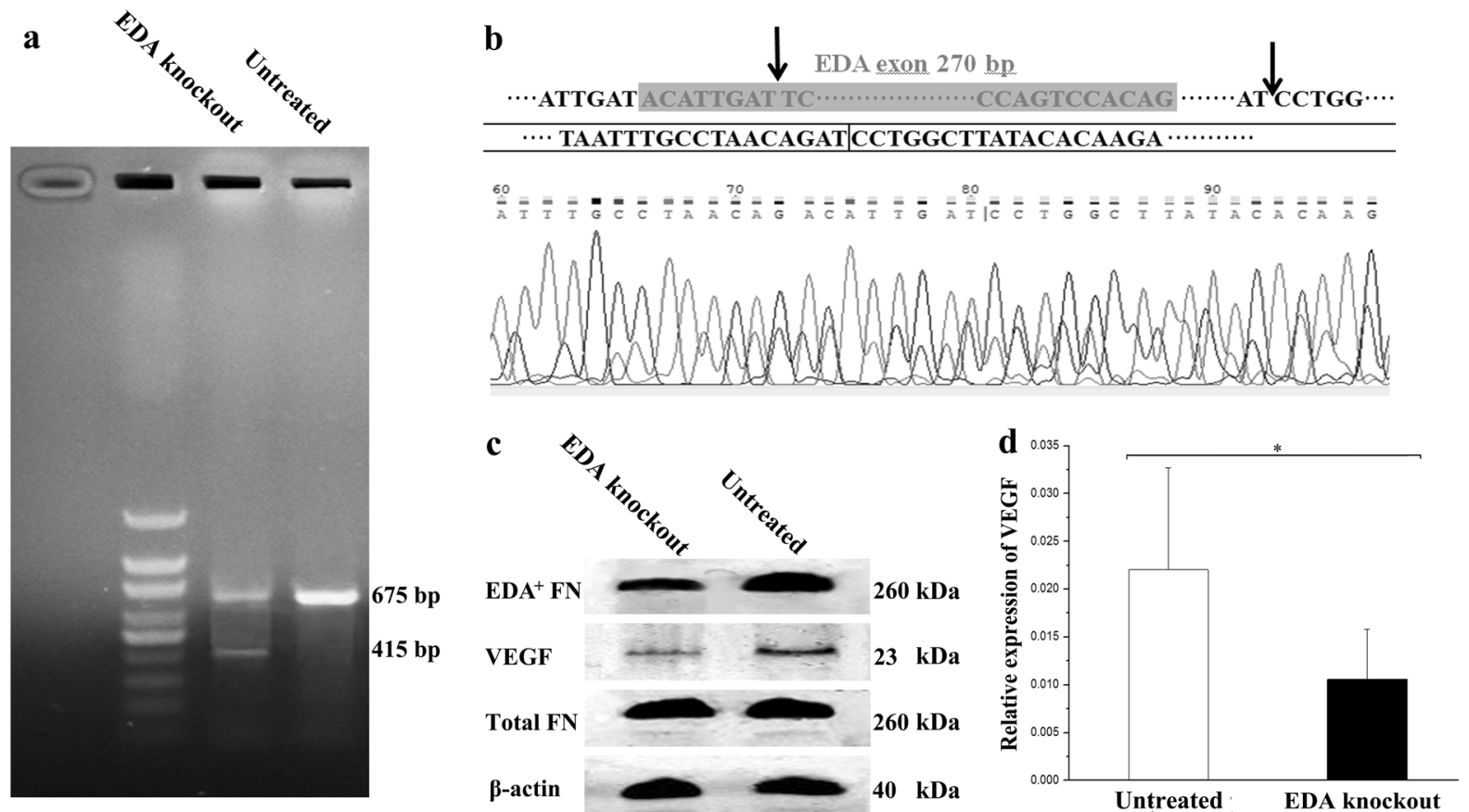

Fig. 3 a The PCR products of the genome isolated from EDA knockout cells, as well as the untreated controls. $\mathbf{b}$ The shorter PCR products (415 bp) representing the NHEJ repaired DSBs in the FN gene were sequenced. $c$ The protein level of EDA ${ }^{+}$FN, total FN, and VEGF in the EDA knockout cells and untreated controls. d The comparison of the relative expression of VEGF between EDA knockout and the untreated fibroblasts $\left({ }^{*} P<0.05\right)$

consists of ECM, cytokines and metabolic products that are involved in the progression of lesions according to our previous studies $[8,20]$. As investigated in the present study, EDA ${ }^{+} \mathrm{FN}$ staining in the fibrous capsule of radicular cysts was positively associated with the radiolucent areas, which were linearly related to the volume of the odontogenic cysts in the jaw bone [27]. Based on this finding, $\mathrm{EDA}^{+} \mathrm{FN}$ exerts osteoclastogenic effects, since the specific peptide motif within the EDA fragment exhibits affinity for receptors located in either preosteoclasts or other stromal cells $[3,5,6]$, leading to direct or indirect effects on osteoclast formation.

According to our investigation, the vessel areas in the fibrous capsules of each hpf were positively associated with both $\mathrm{EDA}^{+} \mathrm{FN}$ staining and the radiolucent area of the cysts. This finding suggested that VEGF expression was induced in the stroma of radicular cysts and perhaps mediated the indirect effects of EDA ${ }^{+} F N$ on osteoclastogenesis, since VEGF is a crucial contributor to vascularization [26] and may serve a substitute for M-CSF during osteoclastogenesis $[10,11]$. Consistent with these findings, relative VEGF expression in fibroblasts from radicular cysts was positively associated with the $\mathrm{EDA}^{+} \mathrm{FN} /$ total $\mathrm{FN}$ ratio, further suggesting an autocrine effect of $\mathrm{EDA}^{+} \mathrm{FN}$ on fibroblasts [3] that subsequently resulted in VEGF production [2]. In contrast to the controls, the EDA ${ }^{+} \mathrm{FN} /$ total $\mathrm{FN}$ ratio was higher in the fibroblasts from the cysts, which was consistent with their increased induction of $\mathrm{Trap}^{+} \mathrm{MNCs}$. The effects of $\mathrm{EDA}^{+} \mathrm{FN}$ on osteoclastogenesis might also be at least partially attributed to VEGF [9], because the positive association between VEGF expression and $\mathrm{Trap}^{+}$MNCs was also observed in fibroblasts from radicular cysts.

In addition, the CRISPR/Cas system was used to knockout the EDA exon in cells from cysts and to elucidate the effects of $\mathrm{EDA}^{+} \mathrm{FN}$ on VEGF expression. As described previously [4], the EDA exon was excluded in some cells, which led to an obvious decrease in $\mathrm{EDA}^{+} \mathrm{FN}$ expression but almost unchanged total FN expression. Simultaneously, the decreased VEGF protein and mRNA levels suggested that its expression depended on the
EDA exon. These findings were consistent with a previous study [2] in which EDA knockout inhibited VEGF expression and subsequent vascularization. Therefore, the specific antibody IST-9 or bevacizumab was added to conditioned medium from cyst fibroblasts to block the interaction between the preosteoclasts and $\mathrm{EDA}^{+} \mathrm{FN}$ or VEGF, respectively $[15,21]$. As illustrated in this study, only bevacizumab inhibited the formation of Trap ${ }^{+}$MNCs to the same extent as the EDA knockout; moreover, this inhibitory effect was almost completely reversed by exogenous rhVEGF. Thus, the effects of $\mathrm{EDA}^{+} \mathrm{FN}$ on osteoclastogenesis were indirectly mediated by VEGF instead of by a direct interaction between $\mathrm{EDA}^{+} \mathrm{FN}$ and preosteoclasts, since the group in which $\mathrm{EDA}^{+} \mathrm{FN}$ was blocked by IST-9 exhibited little inhibitory effect on osteoclastogenesis.

In the present study, $\mathrm{EDA}^{+} \mathrm{FN}$ in the stroma of radicular cysts was positively associated with bone destruction and vascularization, suggesting contributions of EDA and VEGF to osteoclastogenesis. Furthermore, knockout of the EDA exon in the FN gene inhibited VEGF expression in fibroblasts. Therefore, blockade of VEGF by bevacizumab inhibited osteoclastogenesis to the same extent as gene editing of the EDA exon despite the observation that bevacizumab might lead to osteonecrosis $[28,29]$.

\section{ACKNOWLEDGEMENTS}

This study was financially supported by grants from the National Natural Science Foundation of China (No. 81600836) and a China Postdoctoral Science Foundation (No. 2016M591718) and Teaching Reform Foundation of Tongji University (1504104044), and the 10th Excellent Experimental Course Foundation of Tongji University.

\section{AUTHOR CONTRIBUTIONS}

$\mathrm{HCW}$ and $\mathrm{YZ}$ designed the research; HCW performed the experiments; HCW and PW contributed new reagents or analytical tools; HCW, PW, and YWC analyzed the data; and HCW wrote the paper. 
$\mathbf{a}$

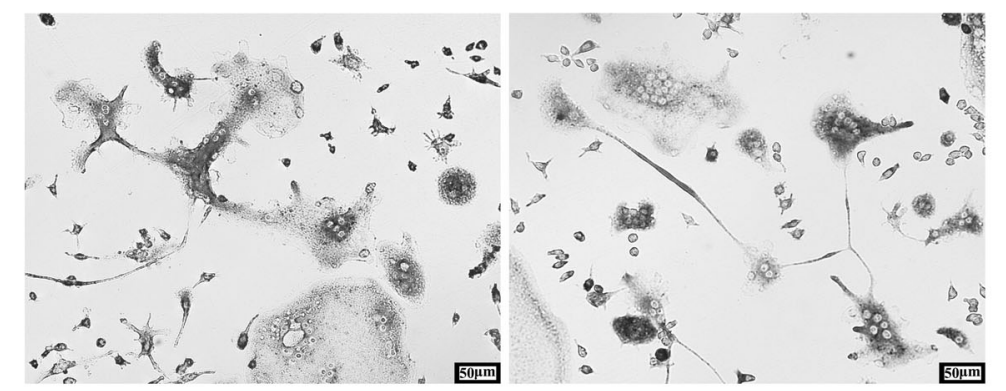

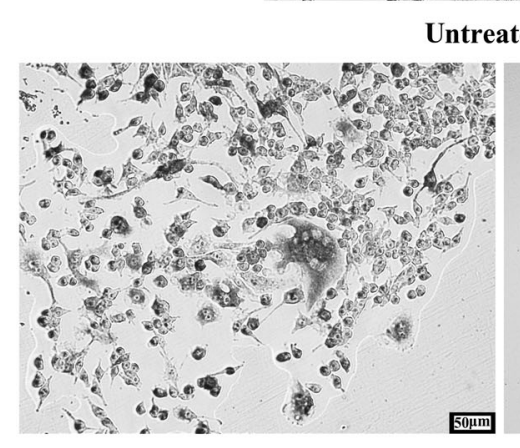

EDA knockout

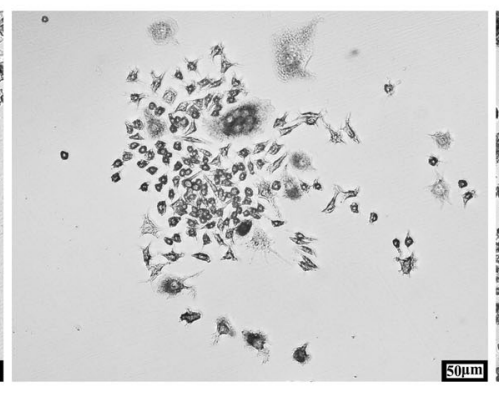

Bevacizumab
$\mathrm{EDA}^{+}$FN blocked

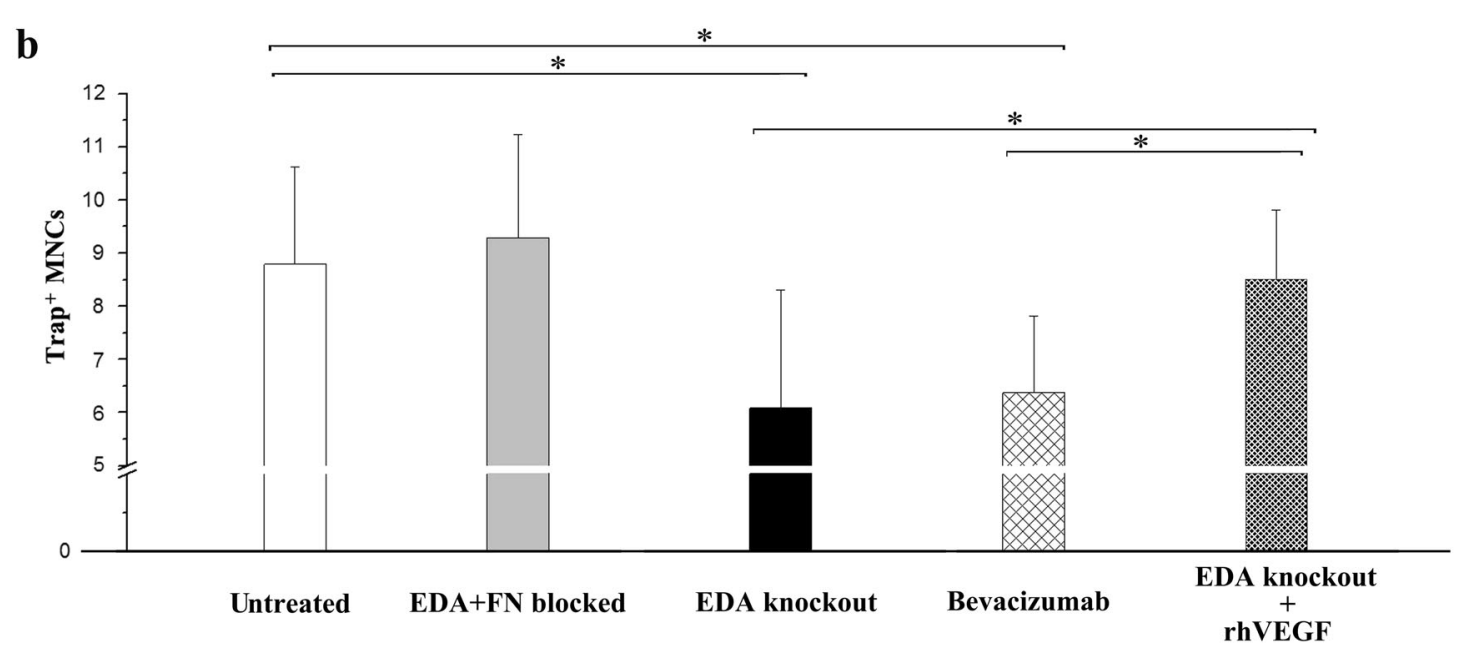

Fig. 4 a The conditioned medium from the fibroblasts of untreated, EDA ${ }^{+}$FN blocked, EDA knockout, bevacizumab, as well as the EDA knockout+rhVEGF groups was used to induce Trap ${ }^{+}$MNCs, respectively (original magnification $\times 200$, scale bar: $50 \mu \mathrm{m}$ ). $\mathbf{b}$ The comparison of the number of Trap ${ }^{+}$MNCs induced by the five groups mentioned above $\left({ }^{*} P<0.05\right)$

Table 4. The concentration of VEGF in the conditioned medium of fibroblasts from cysts

\begin{tabular}{llll}
\hline VEGF $(\mathrm{pg} / \mathrm{mL})$ & Untreated & EDA knockout & $P$-value (on total) \\
\hline Sample 1 & $328.071 \pm 8.384$ & $105.057 \pm 10.470$ & \\
Sample 2 & $531.854 \pm 12.059$ & $104.996 \pm 7.811$ & \\
Sample 3 & $385.090 \pm 3.115$ & $105.369 \pm 2.725$ & $* * 0.001$ \\
Sample 4 & $355.992 \pm 5.170$ & $118.615 \pm 2.695$ & \\
Sample 5 & $432.652 \pm 3.326$ & $129.748 \pm 6.050$ & \\
Sample 6 & $272.061 \pm 2.228$ & $77.926 \pm 6.820$ & \\
\hline${ }^{*} P<0.01$ & & & \\
\hline
\end{tabular}

\section{ADDITIONAL INFORMATION}

Competing interests: The authors declare no competing interests.

\section{REFERENCES}

1. Lemanska-Perek A, Krzyzanowska-Golab D, Pupek M, Klimeczek P, Witkiewicz W, Katnik-Prastowska I. Analysis of soluble molecular fibronectin-fibrin complexes and EDA-fibronectin concentration in plasma of patients with atherosclerosis. Inflammation. 2016;39:1059-68.

2. Lv WQ, Wang HC, Peng J, Wang YX, Jiang JH, Li CY. Gene editing of the extra domain A positive fibronectin in various tumors, amplified the effects of CRISPR/ Cas system on the inhibition of tumor progression. Oncotarget. 2017;8:105020-36.

3. Shinde AV, Kelsh R, Peters JH, Sekiguchi K, Van De Water L, McKeown-Longo PJ. The alpha4beta1 integrin and the EDA domain of fibronectin regulate a profibrotic phenotype in dermal fibroblasts. Matrix Biol. 2015;41:26-35.

4. Wang HC, Yang Y, Xu SY, Peng J, Jiang JH, Li CY. The CRISPR/Cas system inhibited the pro-oncogenic effects of alternatively spliced fibronectin extra domain $A$ via editing the genome in salivary adenoid cystic carcinoma cells. Oral Dis. 2015;21:608-18.

5. Shinde AV, Bystroff $C$, Wang C, Vogelezang MG, Vincent PA, Hynes RO, et al. Identification of the peptide sequences within the EIIIA (EDA) segment of fibronectin that mediate integrin alpha9beta1-dependent cellular activities. J Biol Chem. 2008;283:2858-70. 
6. Gondokaryono SP, Ushio H, Niyonsaba F, Hara M, Takenaka H, Jayawardana ST, et al. The extra domain $\mathrm{A}$ of fibronectin stimulates murine mast cells via toll-like receptor 4. J Leukoc Biol. 2007;82:657-65.

7. Xiang L, Xie G, Ou J, Wei X, Pan F, Liang H. The extra domain A of fibronectin increases VEGF-C expression in colorectal carcinoma involving the PI3K/AKT signaling pathway. PLoS One. 2012;7:e35378.

8. Jiang WP, Sima ZH, Wang HC, Zhang JY, Sun LS, Chen F, et al. Identification of the involvement of LOXL4 in generation of keratocystic odontogenic tumors by RNASeq analysis. Int J Oral Sci. 2014;6:31-38.

9. Wang HC, Li TJ. The growth and osteoclastogenic effects of fibroblasts isolated from keratocystic odontogenic tumor. Oral Dis. 2013;19:162-8.

10. Taylor RM, Kashima TG, Knowles HJ, Athanasou NA. VEGF, FLT3 ligand, PIGF and HGF can substitute for M-CSF to induce human osteoclast formation: implications for giant cell tumour pathobiology. Lab Invest. 2012;92:1398-406.

11. Niida $S$, Kaku M, Amano $H$, Yoshida $H$, Kataoka $H$, Nishikawa $S$, et al. Vascular endothelial growth factor can substitute for macrophage colony-stimulating factor in the support of osteoclastic bone resorption. J Exp Med. 1999;190:293-8.

12. Johnson NR, Savage NW, Kazoullis S, Batstone MD. A prospective epidemiological study for odontogenic and non-odontogenic lesions of the maxilla and mandible in Queensland. Oral Surg Oral Med Oral Pathol Oral Radiol. 2013;115:515-22.

13. Heikinheimo K, Morgan PR, Happonen RP, Stenman G, Virtanen I. Distribution of extracellular matrix proteins in odontogenic tumours and developing teeth. Virchows Arch B Cell Pathol Incl Mol Pathol. 1991;61:101-9.

14. Helmrich U, Di Maggio N, Guven S, Groppa E, Melly L, Largo RD, et al. Osteogenic graft vascularization and bone resorption by VEGF-expressing human mesenchymal progenitors. Biomaterials. 2013;34:5025-35.

15. Canavese M, Ngo DT, Maddern GJ, Hardingham JE, Price TJ, Hauben E. Biology and therapeutic implications of VEGF-A splice isoforms and single-nucleotide polymorphisms in colorectal cancer. Int J Cancer. 2017;140:2183-91.

16. Shim G, Kim D, Park GT, Jin H, Suh SK, Oh YK. Therapeutic gene editing: delivery and regulatory perspectives. Acta Pharmacol Sin. 2017;38:738-53.

17. Zhang H, Zhang L, Chen L, Li W, Li F, Chen Q. Stromal cell-derived factor-1 and its receptor CXCR4 are upregulated expression in degenerated intervertebral discs. Int J Med Sci. 2014;11:240-5.
18. Gadbail AR, Hande A, Chaudhary M, Nikam A, Gawande M, Patil S, et al. Tumor angiogenesis in keratocystic odontogenic tumor assessed by using CD-105 antigen. J Oral Pathol Med. 2011;40:263-9.

19. Persson RE, Tzannetou S, Feloutzis AG, Bragger U, Persson GR, Lang NP. Comparison between panoramic and intra-oral radiographs for the assessment of alveolar bone levels in a periodontal maintenance population. J Clin Periodontol. 2003;30:833-9.

20. Wang HC, Jiang WP, Sima $\mathrm{ZH}$, Li TJ. Fibroblasts isolated from a keratocystic odontogenic tumor promote osteoclastogenesis in vitro via interaction with epithelial cells. Oral Dis. 2015;21:170-7.

21. Kohan M, Muro AF, White ES, Berkman N. EDA-containing cellular fibronectin induces fibroblast differentiation through binding to alpha4beta7 integrin receptor and MAPK/Erk 1/2-dependent signaling. FASEB J. 2010;24:4503-12.

22. Kohen MC, Tatlipinar S, Cumbul A, Uslu U. The effects of bevacizumab treatment in a rat model of retinal ischemia and perfusion injury. Mol Vis. 2018;24:239-50.

23. Miao ZH, Feng JM, Ding J. Newly discovered angiogenesis inhibitors and their mechanisms of action. Acta Pharmacol Sin. 2012;33:1103-11.

24. Garcia CC, Sempere FV, Diago MP, Bowen EM. The post-endodontic periapical lesion: histologic and etiopathogenic aspects. Med Oral Patol Oral Cir Bucal. 2007;12:E585-90.

25. de Oliveira MD, de Miranda JL, de Amorim RF, de Souza LB, de Almeida Freitas R. Tenascin and fibronectin expression in odontogenic cysts. J Oral Pathol Med. 2004;33:354-9.

26. Ramasamy SK, Kusumbe AP, Itkin T, Gur-Cohen S, Lapidot T, Adams RH. Regulation of hematopoiesis and osteogenesis by blood vessel-derived signals. Annu Rev Cell Dev Biol. 2016:32:649-75.

27. Kubota Y, Yamashiro T, Oka S, Ninomiya T, Ogata S, Shirasuna K. Relation between size of odontogenic jaw cysts and the pressure of fluid within. $\mathrm{Br} \mathrm{J}$ Oral Maxillofac Surg. 2004;42:391-5.

28. Hernandez M, Phulpin B, Mansuy L, Droz D. Use of new targeted cancer therapies in children: effects on dental development and risk of jaw osteonecrosis: a review. J Oral Pathol Med. 2017;46:321-6.

29. Anwikar SR, Bandekar MS, Patel TK, Patel PB, Kshirsagar NA. Tetany: possible adverse effect of bevacizumab. Indian J Cancer. 2011;48:31-33. 